\title{
Raman and IR-ATR spectroscopy studies of heteroepitaxial structures with a GaN:C top layer
}

\author{
M. F. Cerqueira ${ }^{1}$, L. G. Vieira ${ }^{1}$, A. Alves $^{2}$, R. Correia $^{2}$, M. Huber ${ }^{3,4}$, A. Andreev ${ }^{3}$, A. Bonanni ${ }^{4}$, \\ M. I. Vasilevskiy ${ }^{1,5}$ \\ ${ }^{1}$ Centro \& Departamento de Física; Universidade do Minho, Campus de Gualtar 4710-057 Braga, Portugal \\ ${ }^{2}$ Departamento de Física e I3N, Universidade de Aveiro, 3710-193 Aveiro, Portugal \\ ${ }^{3}$ Infineon Technologies Austria AG, Siemensstraße 2, 9500 Villach, Austria \\ ${ }^{4}$ Johannes Kepler University Linz, Altenbergerstraße 69, 4040 Linz, Austria \\ ${ }^{5}$ QuantaLab, Universidade do Minho, Braga, Portugal \\ Corresponding author: mikhail@ fisica.uminho.ptl
}

\begin{abstract}
Raman spectroscopy, with both resonant and non-resonant excitation, and infra-red (IR) spectroscopy, in the attenuated total reflection (ATR) configuration, was employed to study lattice vibration modes in a set of carbon-doped $\mathrm{GaN}(\mathrm{GaN}: \mathrm{C})$ epilayers grown by metalorganic vapour phase epitaxy (MOVPE). We analyse Raman and IR-ATR spectra from the point of view of possible effects of the carbon doping, namely: (i) local vibration mode of $\mathrm{C}$ atom in a $\mathrm{N}$ sublattice (whose frequency we theoretically estimate as $768 \mathrm{~cm}^{-1}$ using an isotope defect model), and (ii) shift in the positions of longitudinal modes owing to the phonon-plasmon coupling. We find only some indirect hints of the doping effect on the resonant Raman spectra. However, we show theoretically and confirm experimentally that the IR-ATR spectroscopy can be a much more sensitive tool for this purpose, at least for the considered structures. A weak perturbation of the dielectric function of $\mathrm{GaN}: \mathrm{C}$, caused by the substitutional carbon impurity, is shown to produce a measurable dip in the ATR reflectivity spectra at $\approx 770 \mathrm{~cm}^{-1}$, for both $p$ - and $s$-polarizations. Moreover, it influences a specific (guided-wave-type) mode observed at $\approx 737 \mathrm{~cm}^{-1}$, originating from the GaN layer, which appears in the narrow frequency window where the real parts of the two components of the dielectric tensor of the hexagonal crystal have the opposite signs. This interpretation is supported by our modelling of the whole multilayer structure using a transfer matrix formalism.
\end{abstract}

\section{Introduction}

Gallium nitride $(\mathrm{GaN})$ is a very important semiconductor material because it is the basis for optoelectronic devices operating in the blue range and white light LEDs [1]. The work leading to the development of the technology of this material, in the 1990s, was highlighted with the Nobel Prize in Physics in 2014. The investigation of the properties of GaN and related materials, in particular, new doping possibilities (a necessary process to make LEDs), continues to be active because there are large companies interested in improving the technology and explore new applications. In particular, 
doping with carbon is attractive as a method of achieving $p$-type conductivity of GaN (which usually grows unintentionally $n$-type doped), compatible with the use of silicon substrates and therefore with Si-based electronics [2]. More than 10 years ago this possibility was demonstrated for the first time [3], however, the doping behavior of $\mathrm{C}$ atoms in $\mathrm{GaN}$ crystal still is a matter of debate. In order to achieve $p$-type doping of $\mathrm{GaN}, \mathrm{C}$ atoms should occupy neither cation lattice sites nor interstitial positions but be located in the anion sublattice sites [4] because it would produce an autocompensation effect [2]. If succeeded, it would pave the way towards high-power devices (e.g. highpower high-electron-mobility transistors) that can be integrated into Si-based electronic circuits.

The assessment of the doping behavior of carbon in GaN by means of direct methods (such as Hall effect measurements) offers some interpretation difficulties and the results are not completely conclusive. In fact, many published reports witness that $\mathrm{GaN}: \mathrm{C}$ samples do not exhibit p-type conduction but are semi-insulating [5], which may be the auto-compensation effect. Another possibility to gain information concerning the preferred positions occupied by carbon atoms in the crystal lattice of GaN epilayers, which consists in looking at the lattice vibration modes that can be affected by the presence of electrically active or inactive carbon atoms [4]. In fact, at least three possible effects can be indicated: (i) appearance of local vibration mode(s) produced by carbon atoms (note that interstitial atoms usually are weakly linked to the lattice and their low-frequency vibrations are not detectable in practice), (ii) renormalization of the frequencies of longitudinal optical phonon modes when the concentration of free carriers is sufficiently high, or, in other words, formation of coupled plasmon-phonon modes, which has been measured experimentally for a number of heavily doped polar semiconductor materials including n-GaN [6], and (iii) impurityinduced broadening of the phonon modes detected by means of Raman and infrared (IR) spectroscopies. While the latter is not quite informative, the first two effects, if present, can provide, at least, qualitative information concerning preferred location of carbon atoms in the GaN crystal lattice, and this is the scope of the present work. Raman spectroscopies, with both resonant and nonresonant excitation were employed to study lattice vibration modes in a set of $\mathrm{GaN}$ : $\mathrm{C}$ epilayers grown by metalorganic vapour phase epitaxy (MOVPE) and here we present and discuss the obtained results. As known, IR spectroscopy can provide additional and complementary information concerning phonon or coupled phonon-plasmon modes, for instance, transmittance measurements at oblique incidence provide both TO and LO modes and their interpretation is somewhat simpler than in the case of Raman spectroscopy for uniaxial crystals [7]. Because of the relative complexity of the MOVPE grown multilayer structures (see Fig. 1) we opted for using the attenuated total reflection (ATR) configuration where only a near-surface region of the structure is probed by the evanescent electromagnetic wave created in this method [8]. The interpretation of ATR spectra is more involving (and interesting) and it is also presented in this paper. 


\section{Experimental details}

The samples studied in this work were epitaxial heterostructures with (0001) surface, grown in a MOVPE AIXTRON 6" planetary reactor, with the uppermost GaN layer containing $10^{18}-10^{19}$ carbon atoms per $\mathrm{cm}^{3}$ as determined by Secondary Ion Mass Spectrometry (SIMS). The GaN layer was deposited on a highly resistive AlN/AlGaN multilayer structure grown directly on a single Si crystal substrate, as shown in Figure 1. More details can be found in Ref. 2.

Non-resonant Raman spectroscopy studies were performed using an Alpha300 R confocal Raman microscope (WITec) and a $532 \mathrm{~nm} \mathrm{Nd:YAG} \mathrm{laser} \mathrm{for} \mathrm{excitation.} \mathrm{The} \mathrm{spectra} \mathrm{were} \mathrm{measured} \mathrm{at} \mathrm{room}$ temperature (RT), in a backscattering geometry (laser power $1.5 \mathrm{~mW} ; 100 \mathrm{x}$ Obj; $1800 \mathrm{~g} / \mathrm{mm} ; 2 \mathrm{~s} ; 20$ acquisitions, no polarisers were used). Resonant Raman spectra were measured at RT using a JobinYvon LabRaman HR spectrometer equipped with a multi-channel air cooled $(-70 \circ \mathrm{C}) \mathrm{CCD}$ detector, in the backscattering geometry, using the $325 \mathrm{~nm}$ excitation line of a $\mathrm{He}-\mathrm{Cd}$ laser.

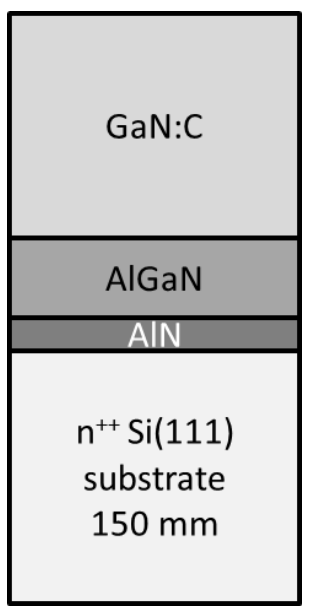

\begin{tabular}{|c|c|c|c|c|}
\hline \multirow{2}{*}{ Sample } & \multicolumn{3}{|c|}{ Thickness (nm) } & \multirow{2}{*}{$\begin{array}{l}\text { C by SIMS } \\
\left.\text { (at. } / \mathrm{cm}^{3}\right)\end{array}$} \\
\hline & AIN & AlGaN & GaN & \\
\hline$\# 1$ & \multirow{3}{*}{120} & \multirow{3}{*}{500} & \multirow{3}{*}{4000} & $2.8 \times 10^{18}$ \\
\hline$\# 2$ & & & & $1.3 \times 10^{19}$ \\
\hline$\# 10$ & & & & $3.5 \times 10^{19}$ \\
\hline
\end{tabular}

Figure 1. Scheme of the multilayer structure and a brief description of three studied samples. The table contains parameters of the structures.

Infrared (IR) spectroscopy in the attenuated total internal reflection (ATR) configuration was performed with a Golden Gate Single Reflection ATR system (Specac) assembled into a Bruker IFS $66 \mathrm{~V}$ spectrometer. The infrared beam was directed onto a prism of monolithic diamond crystal, type IIIA (refractive index $n_{p}=2.146$ ), at an angle of $45^{\circ}$, creating an evanescent wave at the crystal/sample interface. The measurements were performed in vacuum, at room temperature, in the frequency range $550 \mathrm{~cm}^{-1}<\omega<5000 \mathrm{~cm}^{-1}$ with a resolution better than $4 \mathrm{~cm}^{-1}$, by using a globar source, a $\mathrm{KBr}$ beam-splitter and a DTGS detector with $\mathrm{KBr}$ window. Incident light was polarized with a KRS-5 polarizer. The background spectral intensity was acquired when no sample is placed on the prism, so that total reflection occurs. 


\section{Theoretical background}

\section{A. Phonon modes}

A wurtzite structure crystal contains four atoms per unit cell and therefore has nine optical phonon branches. The following low-wavevector $(q \rightarrow 0)$ phonon modes are Raman active [9] in a backscattering geometry: $A_{1}(L O)$ and $E_{2}(h, l)$ for $q \| c ; A_{1}(T O), E_{1}(T O)$ and $E_{2}(h, l)$ for $q \perp c$. (Here $(h, l)$ stands for "high" and "low"). The polar modes of $A_{1}$ and $E_{1}$ symmetries can couple directly to light, i.e. they are IR active. The frequencies of the Raman and IR-active modes in GaN are well known experimentally [4, 7, 10-13] and have also been calculated theoretically [14-16]. These data agree with each other to within $2-3 \mathrm{~cm}^{-1}$. The mode frequencies taken as reference, in this work, are listed in Table I; they were compiled from Refs. [10-16].

Table I. Optical phonon modes of GaN, AlN, and AlGaN (300 K)

\begin{tabular}{|l|l|l|l|l|}
\hline & GaN & AlN & \multicolumn{2}{|l|}{$\mathbf{A l}_{\mathbf{0 . 5}} \mathbf{G a}_{\mathbf{0} .5} \mathbf{N}$} \\
\hline$A_{1}(T O), \mathrm{cm}^{-1}$ & 532 & 610 & $560 ; 605$ & $576.8 ;$ \\
\hline$E_{1}(T O), \mathrm{cm}^{-1}$ & 559 & 669 & $585 ; 640$ & 628.6 \\
\hline$A_{1}(L O), \mathrm{cm}^{-1}$ & 734 & 891 & 835 & \multirow{2}{*}{841.7} \\
\hline$E_{1}(L O), \mathrm{cm}^{-1}$ & 741 & 912 & 845 & \\
\hline$E_{2}(h), \mathrm{cm}^{-1}$ & 570 & 650 & $585 ; 630$ & \multirow{2}{*}{-} \\
\hline$\varepsilon_{z z}^{\infty}\left(\varepsilon_{x x}^{\infty}\right)$ & $5.31(5.14)$ & $4.7(4.5)$ & $5.0(4.8)$ & 4.93 \\
\hline
\end{tabular}

*The values in italics correspond to the isotropic approximation explained in section IIIC.

In contrast, very little is known about local vibration modes of carbon impurities in GaN, A priori, carbon atoms, which are lighter than either nitrogen or gallium atoms, can produce a discrete vibrational mode with a frequency higher than the highest allowed one for the GaN crystal (which is the $E_{1}(L O)$ phonon frequency equal to approximately $\left.740 \mathrm{~cm}^{-1}\right)$ [16]. A numerical estimate of the local vibration frequency of a carbon atom substituting a nitrogen atom, performed using a small cluster approach, yielded values of the order of $745 \mathrm{~cm}^{-1}$ (this mode has a small splitting owing to the hexagonal symmetry of the crystal) [16] and we are not aware of more recent calculated results.

Let us make a simple estimate based on the classical lattice dynamics theory for imperfect crystals [17] that we recently applied to another material [18]. Assuming that a carbon atom inserted into the nitrogen sublattice of $\mathrm{GaN}$ can be modelled as an isotope defect with a mass parameter $\varepsilon=1-M_{C} / M_{N}$ (here $M_{C}$ and $M_{N}$ are the atomic masses), the local harmonic vibration frequency $\left(\omega^{*}\right)$ can be obtained from the equation: 


$$
\varepsilon \omega_{l o c}^{2} \sum_{\sigma}\left|\chi_{\sigma}^{(1)}\right|^{2} \int \frac{g_{\sigma}\left(\omega^{2}\right)}{\omega_{l o c}^{2}-\omega^{2}} d \omega^{2}=1,
$$

where $\sigma$ enumerates branches of the phonon spectrum of the unperturbed crystal, $g_{\sigma}\left(\omega^{2}\right)$ is the density of states (DOS) of the corresponding branch (normalized to unity for each mode) and $\chi_{\sigma}^{(1)}$ is the vibration eigenvalue amplitude on the sublattice where the impurity atom is located (that is, $\left|\chi_{\sigma}^{(1)}\right|^{2}$ is a projection factor of the total DOS onto the said sublattice). For any optical phonon branch it can be estimated as $\left|\chi_{\sigma}^{(1)}\right|^{2}=\left[2\left(1+M_{N} / M_{G a}\right)\right]^{-1}$, since the eigenvectors of optical phonons are inversely proportional to the square root of the atomic mass. The factor of $1 / 2$ accounts for the fact that a GaN unit cell contains two anions (of which only one is substituted by $\mathrm{C}$ ). Then we can introduce an effective defect strength of the cation isotope defect, $\varepsilon^{\prime}=\varepsilon\left|\chi_{\sigma}^{(1)}\right|^{2}$, which yields a value of $\varepsilon_{0}^{\prime} \approx 0.05$ in our case. It represents the purely isotopic defect.

Equation (1) can be solved by making a simple approximation for the unperturbed densities of states (normalized to unity for each branch), for instance, using a linear combination of several Lorentzian functions. With four Lorentzians we have been able to imitate reasonably well the numerically calculated optical phonon DOS for GaN [14-16]. To some extent, the defect strength may be treated as a free parameter meaning that chemical bonds with the impurity atom are not exactly the same as for host atoms. The result of solution of Eq. (1) as a function of $\varepsilon^{\prime}$ is shown in Fig. 2 from which we can see that the local mode appears only above some threshold value of the "defect strength" parameter $\varepsilon^{\prime}$. If we take the value $\varepsilon_{0}^{\prime}$ estimated above, we obtain for the local vibration frequency $\omega_{l o c} \approx 768.5 \mathrm{~cm}^{-1}$. At the same time, the calculation predicts absence of a local mode for carbon in gallium sublattice because $\left|\chi_{\sigma}^{(2)}\right|^{2}=\left[2\left(1+M_{G a} / M_{N}\right)\right]^{-1}<<1$ and $\varepsilon^{\prime}$ lies below the threshold in this case. It is an interesting result because it indicates that probably only carbon atoms substituting nitrogen can originate a local vibration mode.

The local mode, if exists, is expected to be observed by Raman spectroscopy in all symmetry allowed geometries (neglecting its fine structure), although the signal can be weak. The Raman selection rules summarized above apply to non-resonant scattering. Under resonance conditions, the Fröhlich mechanism of electron-phonon interaction whose contribution to Raman scattering is forbidden by the formal selection rules corresponding to phonons with exactly zero momentum $(\vec{q}=0)$, becomes very strong [19]. As a result, one should not expect to see the local mode when real electron-hole pairs are excited by incident photons and the Raman spectrum should be dominated by a strong LO phonon resonance driven by the Fröhlich interaction of the electrons and 
holes with phonons whose wavevectors lie close to (but not exactly at) the $\Gamma$-point. On the other hand, local mode vibrations involving ions should result in dipole moment oscillations and therefore such a mode should perturb the dielectric function of the material (see Fig. 3 and Eq. (9) below).

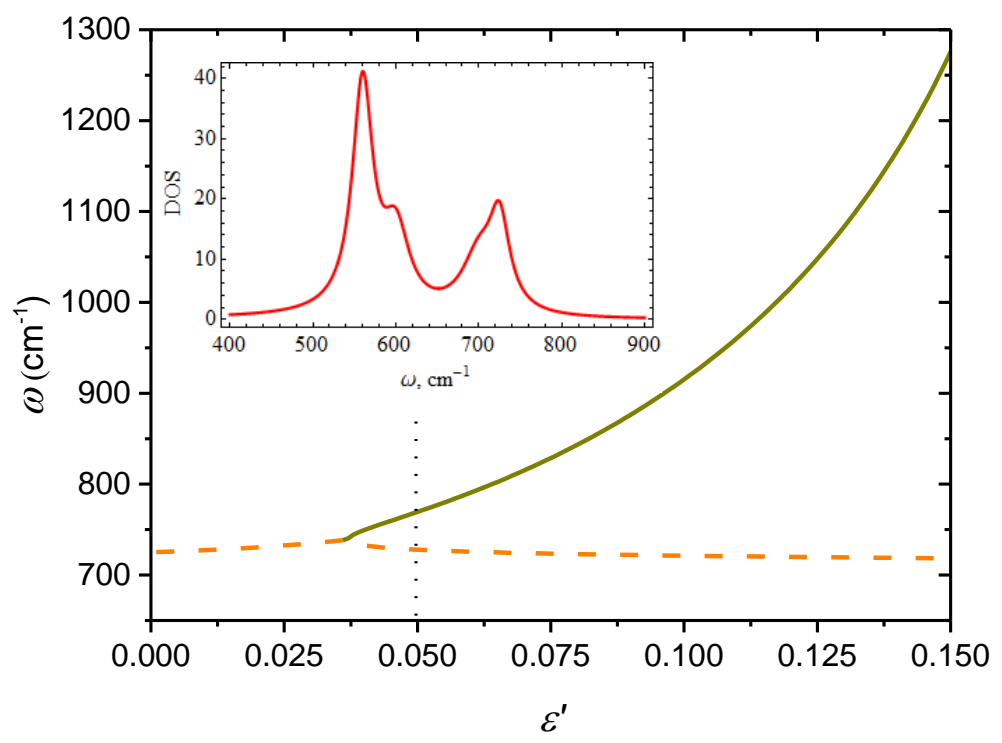

Figure 2. Local mode frequency (full green curve) of a substitution impurity located in $\mathrm{N}$ sublattice of GaN as a function of the defect strength. Another (complex-valued) solution of Eq. (1), the only one below the bifurcation point is shown by the orange dashed line. The vertical dotted line corresponds to $\varepsilon_{0}^{\prime}$ (see text). The inset shows the density of states in the optical phonon region simulated with four Lorentzians, which was used in Eq. (1).

\section{B. Phonon-plasmon coupling}

When the free carrier concentration $(N)$ in a polar semiconductor becomes so high that the plasma frequency,

$$
\omega_{p}=\sqrt{4 \pi e^{2} N /\left(\varepsilon_{\infty} m^{*}\right)}
$$

(where $\varepsilon_{\infty}$ is the high-frequency dielectric constant and $m^{*}$ is the effective mass) approaches the LO phonon frequency, $\omega_{L O}$, the plasmon-phonon coupling takes place and it leads to the appearance of mixed elementary excitations known as plasmon-phonon polaritons. In the small damping regime the frequencies of these excitations are most easily obtained as zeros of the real part of the dielectric function that includes both optical phonon and plasmon contributions,

$$
\varepsilon(\omega)=\varepsilon_{\infty}\left[1+\frac{\omega_{L O}^{2}-\omega_{T O}^{2}}{\omega_{T O}^{2}-\omega^{2}-i \omega_{T O} \Gamma_{T O}}-\frac{\omega_{p}^{2}}{\omega^{2}+i \omega \Gamma_{p}}\right],
$$

where $\omega_{T O}$ is the TO phonon frequency and $\Gamma_{T O}$ and $\Gamma_{p}$ are the phonon and plasmon damping parameters. Equation (3) is valid for the simplest case where only one optical phonon branch exists. In such case, there are two zeros of $\operatorname{Re} \varepsilon(\omega)$ (upper and lower polariton modes). Experimentally, in 
principle, both modes can be observed by either Raman [20] or IR transmission spectroscopy [21] and the modes' positions depend on the doping level. In particular, this effect was observed for $n$ type doped GaN (electron concentrations $n>10^{17} \mathrm{~cm}^{-3}$ ) with an upward shift, a broadening and a decrease of the intensity of the Raman peak originating from the upper phonon-plasmon coupled mode. An appropriate theoretical analysis can yield an estimate of the free carrier concentration.

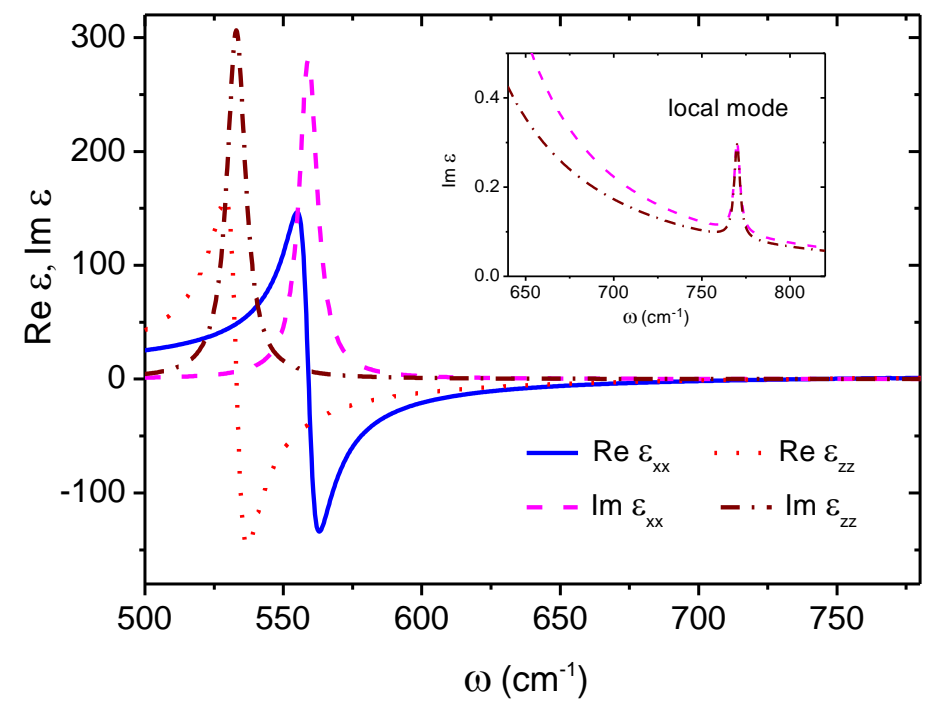

Figure 3. Calculated dielectric functions ( $x x$ and $z z$ components) of GaN. The inset shows the local mode region enlarged. The TO phonon damping parameters are $8 \mathrm{~cm}^{-1}$ for both $\left(\mathrm{A}_{1}\right.$ and $\left.\mathrm{E}_{1}\right)$ modes. The phonon mode frequencies are taken from Table 1. For the local mode in $\mathrm{GaN} \rho_{\text {loc }}=0.0005$ and $\Gamma=4 \mathrm{~cm}^{-1}$ (see Eq. (9)). No plasmon contribution was assumed.

\section{ATR and phonon-polaritons}

What should we expect to find in IR ATR spectra? In contrast with Raman and oblique incidence IR transmission spectra, ATR spectral features do not immediately indicate certain phonon mode frequencies. The evanescent wave coming out of the ATR prism couples only to polar excitations with sufficiently large wavevectors that lie beyond the light cone (and therefore do not interact with usual propagating waves). Examples of such excitations are surface surface phonon-polaritons (SPPs) for a polar dielectric or surface plasmon-polaritons for a metal. The necessary condition for the existence of such waves is that (the real parts of) the dielectric constants of the two interfacing media have opposite signs $[8,22]$. For an interface between vacuum and an isotropic dielectric medium (or semiconductor) it occurs for the frequencies within the so called reststrahlen band $\left(\omega_{T O}<\omega<\omega_{L O}\right)$ and the dispersion relation of the surface phonon-polaritons (SPPs) is well known, $c k_{x}=\omega \sqrt{\varepsilon(\omega) /(\varepsilon(\omega)+1)}$, where $k_{x}$ is the SPP wavevector. A dip in the ATR reflectivity spectrum occur at a frequency for which the SPP dispersion curve crosses the so called ATR scanline, 


$$
c k_{x}=\omega n_{p} \sin \vartheta_{p},
$$

where $\vartheta_{p}$ is the beam incidence angle in the prism. Combining (4) with the above dispersion relation where $\varepsilon(\omega)$ is from Eq. (3) (neglecting the plasma term) yields:

$$
\omega_{* *}=\sqrt{\frac{\omega_{L O}^{2}-\beta \omega_{S}^{2}}{1-\beta}} ; \quad \beta=n_{p}^{2} \sin ^{2} \vartheta_{p}\left(\varepsilon_{\infty}+1\right) / \varepsilon_{\infty},
$$

where $\omega_{S}=\sqrt{\left(\omega_{T O}^{2}+\varepsilon_{\infty} \omega_{L O}^{2}\right) /\left(1+\varepsilon_{\infty}\right)}$ is the surface mode frequency for $k_{x} \rightarrow \infty$.

For a uniaxial material with $c$ axis normal to the surface, the SPP dispersion relation $\omega\left(k_{x}\right)$ follows from the equation $[8,22]$ :

$$
\frac{\varepsilon_{1}}{\sqrt{k_{x}^{2}-\varepsilon_{1} \omega^{2} / c^{2}}}+\frac{\varepsilon_{x x}}{\sqrt{\varepsilon_{x x} k_{x}^{2} / \varepsilon_{z z}-\varepsilon_{x x} \omega^{2} / c^{2}}}=0
$$

where $\varepsilon_{1}=1$ for vacuum and $\varepsilon_{x x}$ and $\varepsilon_{z z}$ are the principal components of the dielectric tensor of the material. Each of them are given by expressions analogous to Eq. (3) with the phonon frequencies of the $A_{1}$ modes for $\varepsilon_{z z}$ and $E_{1}$ modes for $\varepsilon_{x x}$. The real and imaginary parts of the dielectric functions $\varepsilon_{z z}$ and $\varepsilon_{x x}$ are shown in Fig. 3.

The solution of the system of equations (6) and (4) yields $\omega_{*} \approx 700 \mathrm{~cm}^{-1}$ for $n_{p}=2.416$ and $\vartheta_{p}=45^{\circ}$. (For comparison, isotropic approximation and formula (5) give $\omega_{*} \approx 705 \mathrm{~cm}^{-1}$ ). Experimentally, such a mode was observed at $699 \mathrm{~cm}^{-1}$ in ATR studies of wurtzite GaN [23]. Although the $\mathrm{GaN}$ top layer in our structures is quite thick $(4 \mu \mathrm{m})$, the interface with the buffer layer may influence the frequency of the vacuum/GaN surface mode and eventually originate other spectral features (in fact, it does, as we shall see below!). Relatively thin $(\approx 1 \mu \mathrm{m})$ GaN layers on substrates different from AlGaN have been studied by IR-ATR spectroscopy $[24,25]$ and indeed in this case the measured surface mode frequency is somewhat different from that observed in Ref. [23]. To consider the GaN/AlGaN interface, we need to model the dielectric function of the latter. It is well established that the LO phonons of hexagonal AlGaN alloy follow one-mode behaviour while the rest of the Raman or IR active modes exhibit a two-mode behaviour [7]. In terms of phononrelated dielectric function, this situation can be understood as usual two-mode behaviour, however, the oscillator representing the AlN-like mode is rather weak and $\operatorname{Re} \varepsilon(\omega)$ does not attain zero in the region between the GaN-like and AlN-like TO phonon frequencies. Therefore we model the dielectric function of the $\mathrm{Al}_{0.5} \mathrm{Ga}_{0.5} \mathrm{~N}$ alloy, in the isotropic approximation which is sufficient for our purposes, in the following standard way [26]: 


$$
\varepsilon_{A l G a N}(\omega)=\varepsilon_{\infty}\left[1+\sum_{i}^{N} \frac{\rho_{i}\left(\omega_{L O}^{2}-\omega_{T O_{i}}^{2}\right)}{\omega_{T O_{i}}^{2}-\omega^{2}-i \omega_{T O_{i}} \Gamma_{T O_{i}}}\right],
$$

where $N=2$ for $\mathrm{AlGaN}, \Gamma_{T O_{i}}$ is the TO mode damping and $\rho_{i}$ is a dimensionless oscillator strength (a number between 0 and 1 ).

The phonon mode frequencies are presented in Table I where those related to AlN and $\mathrm{Al}_{0.5} \mathrm{Ga}_{0.5} \mathrm{~N}$ were obtained from Fig. 4 of Ref. [27] and the high frequency dielectric constants were taken from Ref. [28]. The second column for the alloy contains the "isotropic" values (obtained in the usual way, i.e. $1 / 3$ of the value corresponding to $c$-axis plus $2 / 3$ of the in-plane value minding that squares of the phonon frequencies are averaged). The dielectric function of the $\mathrm{Al}_{0.5} \mathrm{Ga}_{0.5} \mathrm{~N}$ alloy is shown in Fig. 4 (compare to Fig. 3 for GaN:C). We notice that the real part of it is negative in the broad region from 570 to $810 \mathrm{~cm}^{-1}$ which overlaps with the $\mathrm{GaN}$ reststrahlen band.

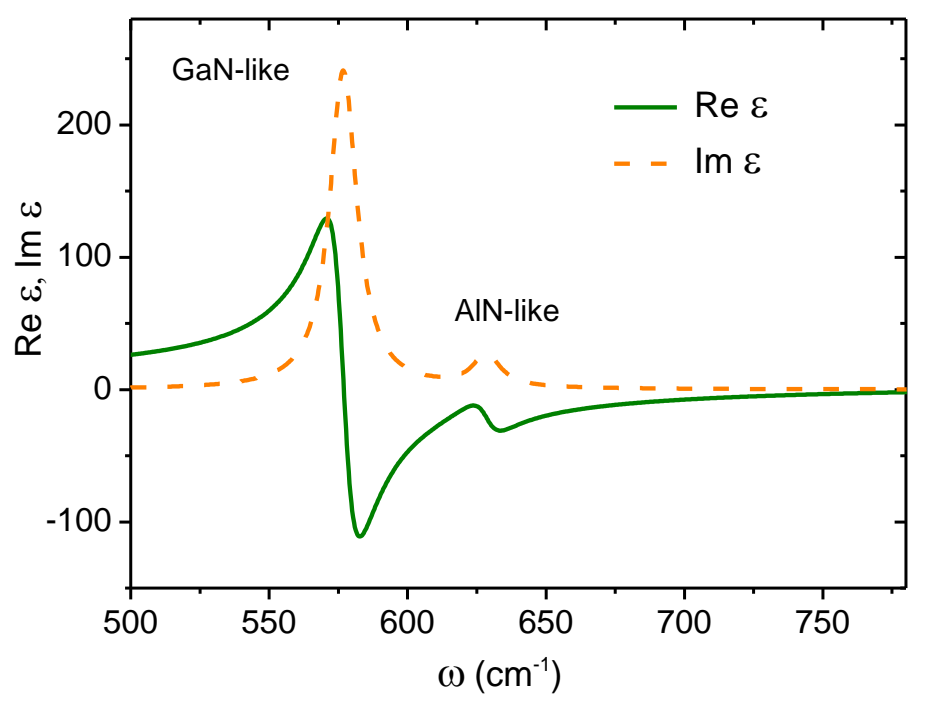

Figure 4. Calculated dielectric function of $\mathrm{Al}_{0.5} \mathrm{Ga}_{0.5} \mathrm{~N}$ (isotropic approximation). The TO phonon damping parameters are $12 \mathrm{~cm}^{-1}$ for both (GaN-like and AlN-like) modes. The oscillator strengths are $\rho_{1}=0.9$ and $\rho_{2}=0.1$.

Polariton modes in a GaN layer cladded by two semi-infinite media (air and AlGaN alloy) can be obtained in a number of ways. We did not find in the literature ready expressions for the case of two unequal cladding media, so we had to do it ourselves and we found it convenient to use the formalism employing the Fresnel coefficients, which link the complex amplitudes of the reflected and transmitted waves to that of the incident electromagnetic wave. It is important to notice that even though we have a uniaxial crystal film, its $c$ axis is normal to the interfaces (i.e. coincides with $z$ axis of the system) and therefore the system has axial symmetry. It means that p- and s-polarized waves are decoupled from each other and can be considered separately. Here we are interested in $p$ polarization. 
The Fresnel coefficients, $\hat{r}^{p}$ and $\hat{t}^{p}$, for a uniaxial slab with $c$ axis normal to the surface are (see Appendix for details):

$$
\begin{aligned}
& \hat{t}^{p}=\frac{2}{\cos \left(p_{2} d\right)\left(1+\frac{\varepsilon_{1} k_{3 z}}{\varepsilon_{3} k_{1 z}}\right)-i \sin \left(p_{2} d\right)\left(\frac{\varepsilon_{x x} k_{3 z}}{\varepsilon_{3} p_{2}}+\frac{\varepsilon_{1} p_{2}}{\varepsilon_{x x} k_{1 z}}\right)} ; \\
& \hat{r}^{p}=\hat{t}^{p}\left[\cos \left(p_{2} d\right)-i \frac{\varepsilon_{x x} k_{3 z}}{\varepsilon_{3} p_{2}} \sin \left(p_{2} d\right)\right]-1,
\end{aligned}
$$

where $k_{1,3 z}=\sqrt{\varepsilon_{1,3} \omega^{2} / c^{2}-k_{x}^{2}}$ and $p_{2} \equiv \sqrt{\varepsilon_{x x} / \varepsilon_{z z}} k_{2 z}=\sqrt{\varepsilon_{x x} \omega^{2} / c^{2}-\varepsilon_{x x} k_{x}^{2} / \varepsilon_{z z}}$. As known [29], surface polaritons yield zeros of the common denominator of $\hat{r}^{p}$ and $\hat{t}^{p}$ (if damping is neglected) when the wavevector components along the $z$ axis are imaginary for all the media constituting the structure, i.e. $k_{1,3 z}=i \kappa_{1,3}$ and $p_{2}=i \kappa_{2}$ (all square roots are to be taken positive). Setting the denominator in (8) equal to zero provides the dispersion relations of SPPs in a GaN layer cladded by two semi-infinite media with dielectric constants $\varepsilon_{1}$ and $\varepsilon_{3}$. It is easy to verify that this relation reduces to Eq. (6) if the film thickness $d \rightarrow \infty$.

In order to determine the frequencies of the ATR spectral features (peaks of absorption or minima of reflectance), we need to substitute $k_{x}(\omega)$ from Eq. (4) into the polariton dispersion relation. Since damping of the phonon modes is important, it is convenient to plot $\operatorname{Im} \hat{t}^{p}$ as a function of frequency and its peaks yield the SPP mode frequencies to be expected in the ATR configuration with $n_{p}=2.416$ and $\vartheta_{p}=45^{\circ}$ (see Fig. 5). We see from Fig. 5 that the vacuum/GaN SPP mode is practically unshifted with respect to its value of $700 \mathrm{~cm}^{-1}$ characteristic of bulk GaN, which is expectable since $\kappa_{2} d$ is large. The other mode $\left(757 \mathrm{~cm}^{-1}\right)$ lies beyond the reststrahlen band of GaN and corresponds to the GaN/AlGaN interface. It is also a surface polariton mode but the role of GaN in this case is inverted compared to the vacuum/GaN interface because the real part of its dielectric function here is positive, while $\operatorname{Re} \varepsilon_{\text {AlGaN }}(\omega)<0$ (see Fig. 4). This feature is analogous to the so called Berreman mode observed in reflection spectra of thin films of polar dielectrics deposited on a metal [30].

As for the third mode seen at $\approx 740 \mathrm{~cm}^{-1}$, with $\operatorname{Im} \hat{t}^{p}<0$, it corresponds to a kind of guided wave because we verify that for it the $z$-component of the wavevector is imaginary in the cladding media, while for the effective wavevector in the GaN layer, $p_{2}$, we have $\operatorname{Re} p_{2}>\operatorname{Im} p_{2}$. This mode is specific of the uniaxial GaN crystal because there is a frequency window between the $A_{1}(L O)$ and $E_{1}(L O)$ phonon modes where $\operatorname{Re} \varepsilon_{x x} / \varepsilon_{z z}<0$. It is precisely in this frequency window that such a 
mode appears and, despite its oscillating nature inside the GaN layer, its frequency does not depend much on the layer thickness, as one might expect for a guided mode. Calculations show that it appears only if the GaN layer thickness is above certain value.

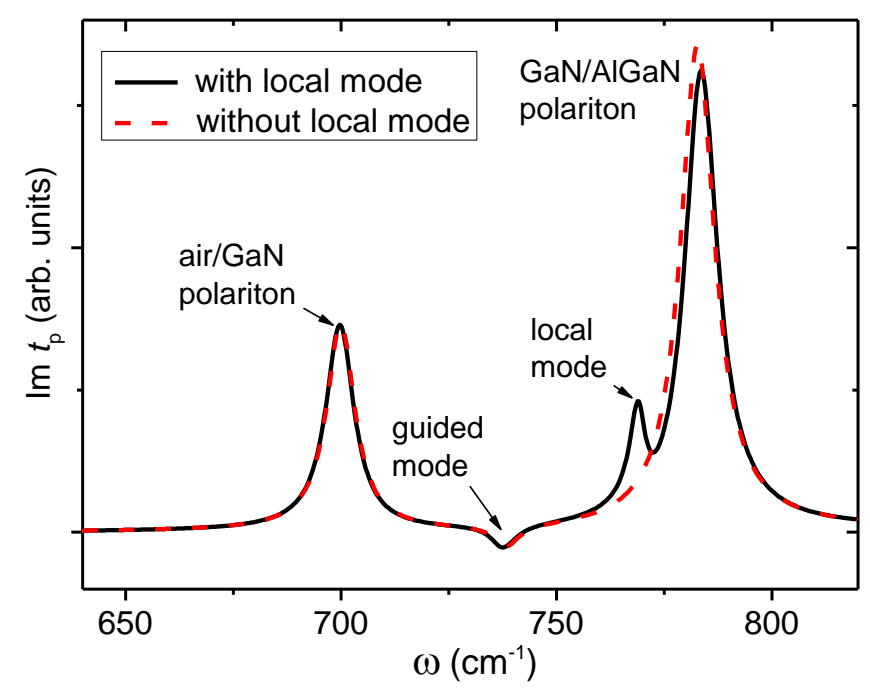

Figure 5. Imaginary part of the Fresnel coefficient calculated for a $4 \mu \mathrm{m}$ thick GaN layer cladded by semi-infinite vacuum and AlGaN media, using Eq. (8) with $k_{x}$ obeying Eq. (4) (ATR scan line). The dielectric function of GaN was used without (dashed line) and with (solid line, $\rho_{\mathrm{loc}}=0.0005$ ) local mode contribution. Other parameters are from Table I.

Finally, what is the expected effect of the carbon local phonon mode (if it indeed exists) in GaN on the ATR spectra? We were unable to find an answer to this question in the literature, so we used the following simple model in order to get it. An additional Lorentzian term was added to both $\varepsilon_{x x}(\omega)$ and $\varepsilon_{z z}(\omega)$,

$$
4 \pi \chi_{l o c}(\omega)=\frac{\rho_{l o c} \varepsilon_{\infty}\left(\omega_{L O}^{2}-\omega_{T O}^{2}\right)}{\omega_{l o c}^{2}-\omega^{2}-i \omega_{l o c} \Gamma_{l o c}},
$$

where $\rho_{l o c}$ is a dimensionless oscillator strength of the local mode of frequency $\omega_{l o c}$. The latter was taken as $770 \mathrm{~cm}^{-1}$, close to the value estimated for the purely isotopic defect from Eq. (1). Despite the weakness of its contribution to the dielectric function seen in Fig. 3, the effect of this additional term on the Fresnel coefficients turns out quite noticeable if $k_{1,3 z}$ are imaginary. It appears in the spectrum of $\operatorname{Im} \hat{t}^{p}$ (Fig. 5) and also in s-polarization. We also notice that the guided wave mode is slightly pushed downwards and the Berreman-type mode is a little bit shifted towards the higher frequencies when the local mode contribution (9) is included.

\section{Results and discussion}




\section{A. Non-resonant Raman spectra}

The spectra shown in Fig. 6 contain two principal peaks assigned to the symmetry-allowed firstorder scattering by lattice vibration modes of a hexagonal crystal [ $E_{2}(h)$ and $A_{1}(L O)$ ] [9]. A weak $E_{2}(l)$ mode at $144 \mathrm{~cm}^{-1}$ was also detected. The low widths (FWHM) of the non-polar $E_{2}$ modes (2 $\mathrm{cm}^{-1}$ for the lower and $5 \mathrm{~cm}^{-1}$ for the upper one for sample \#10) witness the high crystal quality of the GaN epilayers. The polar $A_{1}(L O)\left(733 \mathrm{~cm}^{-1}\right)$ mode is only slightly broader $\left(\mathrm{FWHM} \approx 8 \mathrm{~cm}^{-1}\right)$ and its position does not depend on the doping level and is in agreement with previously published data (Table I). Even though its intensity seems to correlate with the carbon concentration, in fact, its variation between different samples is not very significant when the intensity is normalised to the spectral maximum (see inset in Fig. 6). These observations exclude any significant phonon-plasmon coupling in our samples, which was observed in Ref. [6] for $n$-type doped GaN. In that work, an upward shift, a broadening and a decrease of the intensity of the Raman peak originating from the coupled plasmon-LO-phonon mode was observed for electron concentrations $n>10^{17} \mathrm{~cm}^{-3}$, while nothing like this is seen in our case. Taking the effective mass of holes 6.5 times larger than that of conduction band electrons [13], we can conclude from Eq. (2) that the plasma frequency for holes should be only $\approx 2.5$ times lower than for electrons, for the same concentration. Thus, the plasmonphonon effects for $p$-type doped $\mathrm{GaN}$ should certainly be observable if the hole concentration were at least $10^{18} \mathrm{~cm}^{-3}$, so we conclude that it is bellow this value in our samples.

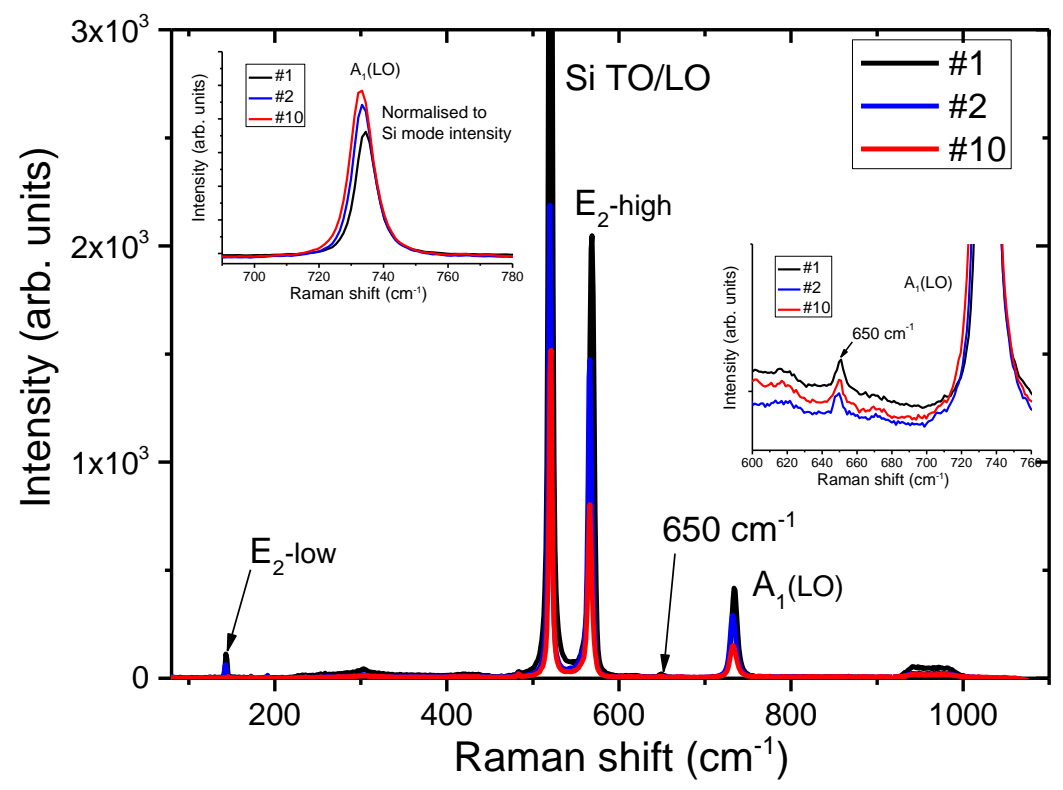

Figure 6. Room temperature Raman spectra of three different doping GaN:C samples (nominal C concentration increases from sample \#1 to \#2 to \#10). The insets show fragments of the spectra enlarging the $A_{1}(L O)$ mode normalised to the Si mode intensity (left) and a weak extra mode at $\approx 650 \mathrm{~cm}^{-1}$ (right).. Excitation wavelength $532 \mathrm{~nm}$. 
Beyond the "proper" GaN modes, we also observed: (i) a very strong peak located at $\approx 520 \mathrm{~cm}^{-1}$, and (ii) an extra mode at $\approx 650 \mathrm{~cm}^{-1}$, much weaker in intensity and seen for all GaN:C samples studied (inset in Fig. 6). The former was verified to originate from the silicon substrate. Owing to the high transparency of the heterostructure to the $532 \mathrm{~nm}$ light and the large thickness of the silicon substrate, this mode caused by Si LO/TO phonons dominates the Raman spectrum (while it is absent under resonant conditions, see below). Concerning the weak peak (ii), it might be related to a local vibration mode of the carbon impurity (more likely in the nitrogen sublattice, according to its frequency) and we are carrying on further investigation in order to understand the origin and relevance of this mode to the scope of this study. However, it looks plausible that it may be related to the AlN-like $E_{2}(h)$ mode of the underlying AlGaN layer. Indeed, such a mode has been observed in AlGaN alloys with Al fraction exceeding 0.6 [6, 27]. In Ref. [27] its existence is assumed also for lower $\mathrm{Al}$ fraction and linear extrapolation yields a frequency somewhat below $650 \mathrm{~cm}^{-1}$ for $\mathrm{Al}_{0.5} \mathrm{Ga}_{0.5} \mathrm{~N}$.

\section{B. Resonant Raman spectra}

Raman spectroscopy results obtained under UV (resonant) excitation for different $\mathrm{C}$ doping of $\mathrm{GaN}$ :C thin films are presented in Fig. 7. Although the spectra contain LO phonon-related features up to the 5-th order [11], they are dominated by the strong exciton photoluminescence (PL) band which is commonly observed in $\mathrm{GaN}$ crystals with different doping characteristics, located at $\approx 3.4$ $\mathrm{eV}$ at room temperature [31], as it is the case of our spectra. In contrast with the non-resonant spectra of Fig. 6, in which only one phonon-related feature is seen, which is designated as LO mode. As mentioned in Sec. IIIA, the (forbidden in the dipole approximation) interaction of excitons created under resonant excitation with longitudinal optical phonons with small but finite wavevector becomes so strong that the symmetry-dictated formal selection rules become irrelevant [19]. Phonons with finite $q$ can be considered as a linear combination of $A_{1}(L O)$ and $E_{1}(L O)$ modes of the $\Gamma$ point, with an intermediate frequency [9].

Resonant Raman spectroscopy (RRS) data indicate that the carbon doping suppresses exciton PL (compare the spectra of the three samples in Fig. 7). This is a common phenomenon and it does not necessarily mean that the impurity is electrically active. The incorporation of impurities can simply increase the nonradiative recombination rate even if the impurities produce very deep levels and are in an electrically inactive state. Indeed, the importance of the trapping process has been demonstrated for $\mathrm{GaN}$ and it is likely to be directly related to $\mathrm{C}$ based defects [32]. Moreover, electron transitions from the conduction band to defect or impurity states can cause characteristic photoluminescence, such as yellow and green emission bands in $\mathrm{GaN}$ [31-33], which have been attributed to $\mathrm{C}_{\mathrm{N}} \mathrm{O}_{\mathrm{N}}$ complexes [32] and isolated $\mathrm{C}_{\mathrm{N}}$ atoms [33], respectively. It is evident that each of these concurrent 
(defect-mediated radiative and nonradiative) mechanisms decreases the intensity of the exciton PL in the ultraviolet (UV) range. A more detailed discussin of the PL emission is beyond the scope of this work. Let us just notice that there is some decrease in the excitation transition energy with doping since the centre of the PL band shifts to the right from sample \#1 to \#10 (Fig. 7). We may suggest that the UV band observed in our RRS spectra is composed of many lines originating mostly from excitons bound to impurities and their complexes, at least for the heavier doped samples \#2 and \#10. A plethora of such emission lines have been observed in detailed low-temperature studies of GaN crystals [31].

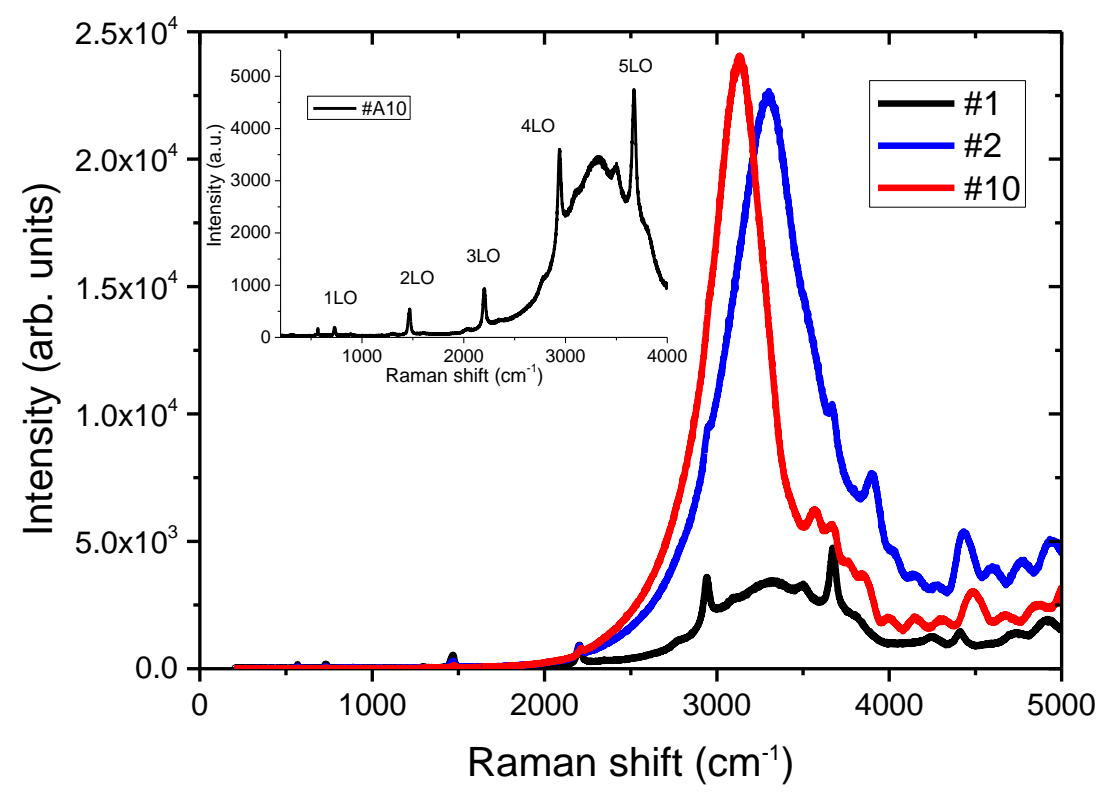

Figure 7. Room temperature Raman spectra of three GaN:C samples with different doping levels. Inset: Detail of the phonon-related features up to the 5-th order. Excitation wavelength $325 \mathrm{~nm}$.

The unequal separations between the subsequent LO peaks make us think that they do not represent the true multiphonon Raman effect (i.e. scattering with the simultaneous creation of several optical phonons). As discussed in Ref. [34] in relation to similar spectra of doped $\mathrm{ZnO}$ films, the observed multiple LO phonon "resonant Raman effect", in fact, is a sequence of (phonon-assisted) indirect processes of light absorption and emission by excitons. Understanding of the RRS spectra in terms of the previously published cascade model [35] implies that the carbon doping does not decrease the exciton-phonon interaction, as one might expect if the impurity provided additional charge carriers that would cause a strong screening of the Fröhlich interaction between the longitudinal optical phonon and the exciton.

Although these Raman results and electrical measurements [2] suggest that carbon doping does not provide the desired high concentration of free carriers, it is not possible to tell whether it is due to that the fact that the majority of the carbon atoms are in an electrical inactive-state or there is a 
strong self-compensation effect. Our theoretical estimates (Sec. IIIA) suggest that carbon substituting gallium should not produce a local vibration mode, therefore it cannot be detected by any lattice vibration spectroscopy method. Yet, as we shall see in the next section, IR-ATR spectroscopy can clearly reveal the local vibration mode due to carbon atoms in the nitrogen sublattice.

\section{IR-ATR spectra}

Figure 8 shows the relevant frequency domain part of the ATR spectra for the three analysed samples using polarized light. As expected, the s-polarized spectra are essentially featureless with the reflectivity close to unity because in the shown spectral region no phonon-polaritons exist in this polarization. The $p$-polarized spectra in Fig. 8 do show features that should be expected from our analysis in Sec. IIIC, related to (i) SPP mode at the sample surface (broad band centred at 620-630 $\mathrm{cm}^{-1}$ ) and (ii) the guided wave mode at the GaN/AlGaN interface (dip at $\approx 737 \mathrm{~cm}^{-1}$ ). Also, a small but clear minimum (iii) is seen in both polarizations for the most heavily doped sample \#10.

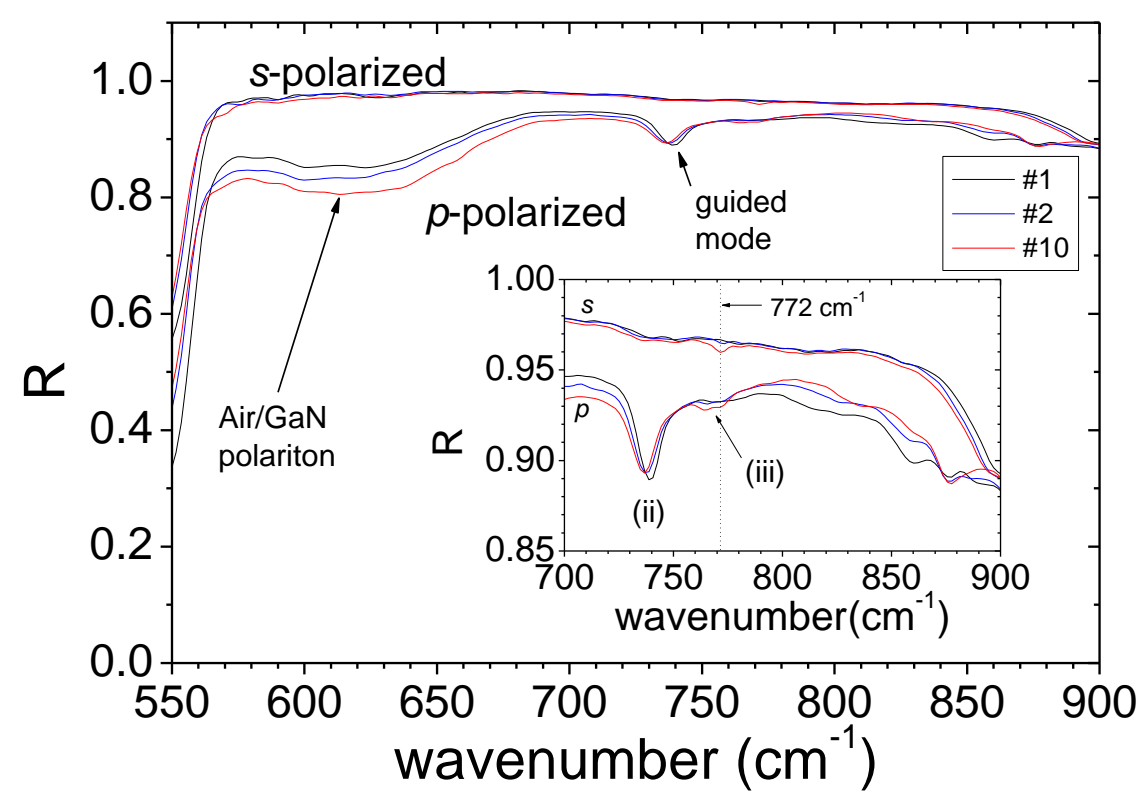

Figure 8. Experimental $s$ - and $p$-polarized IR ATR spectra of three GaN:C samples with different doping levels. Inset shows a fragment of the spectra in the range of the surface and interface polaritons.

The vacuum/GaN SPP mode (i)=is unexpectedly broad and appears considerably lower in frequency than predicted for the model considering the GaN layer cladded by semi-infinite vacuum and AlGaN media in Sec. IIIC (see Fig. 5). In order to clarify this point, we undertook a direct modelling of the ATR spectra of the whole structure (including the prism and the substrate, which were treated as semi-infinite media). They were calculated using a general $4 \times 4$ transfer matrix method [36] (see Appendix for details). The dielectric functions of GaN, $\varepsilon_{z z}$ and $\varepsilon_{x x}$, were taken with the local mode 
contribution (9) included (the parameters are given in the caption of Fig. 3), while for AlGaN we used expression (7) with $N=2$ to describe the two mode behaviour and evaluate both $\varepsilon_{z z}$ and $\varepsilon_{x x}=\varepsilon_{y y}$ considering $\rho_{1}=0.9, \rho_{2}=0.1$ and the parameters of Table I (here 1 and 2 refer to the modes in the table in the order of their appearance). The dielectric function of silicon substrate was described by a Drude model $\left(\varepsilon=\varepsilon_{\infty}-\omega_{p}^{2} /\left(\omega^{2}+i \gamma \omega\right), \varepsilon_{\infty}=11.7, \omega_{p}=585 \mathrm{~cm}^{-1}, \gamma=5 \mathrm{~cm}^{-1}\right)$.

Our investigation shows that the reason for the wide width of the vacuum/GaN SPP mode is the small thickness of the vacuum gap between the base of the ATR prism and the GaN surface. The results of modelling of the reflectivity spectra of the whole structure including the prism, shown in Fig. 9, demonstrates how this mode is affected by the vacuum gap thickness, $d_{g a p}$. The calculated ATR reflectivity spectrum presented in the main panel of Fig. 9, obtained with $d_{\text {gap }}=$ of $300 \mathrm{~nm}$, reproduces the broadened and downshifted vacuum/GaN polariton mode quite nicely. The inset of Fig. 9 shows that the "intrinsic" SPP frequency of $\approx 700 \mathrm{~cm}^{-1}$ is attained only for $d_{\text {gap }} \approx 6 \mu \mathrm{m}$. The intermediate value of the gap thickness $(\approx 0.3 \mu \mathrm{m}) \quad$ is a compromise when surface polariotn modes are not too strongly deviated from their intrinsic positions and the local vibration mode is already clearly seen.

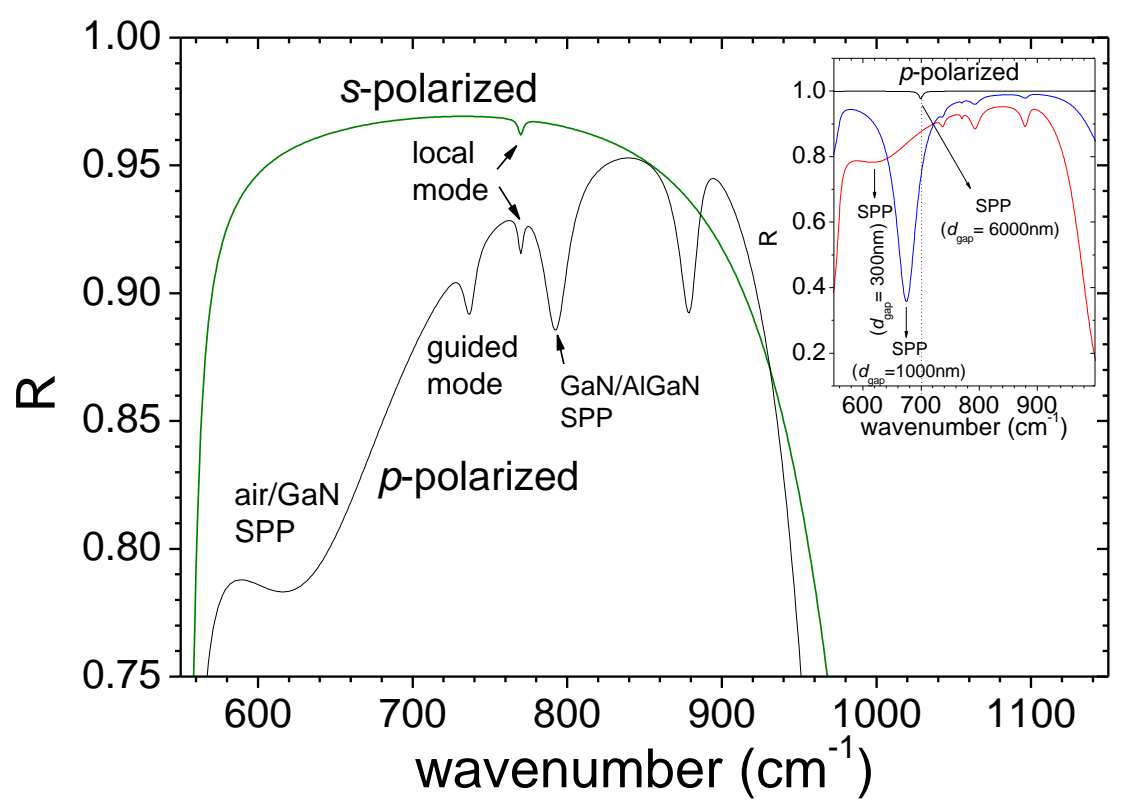

Figure 9. Calculated $s$ - and $p$-polarized ATR spectra for the whole structure of Fig. 1 (GaN/AlGaN/AlN/Si). The inset shows the effect of the thickness of the vacuum gap between the ATR prism and the structure on the SPP mode. Here we used $\rho_{\text {loc }}=0.00005$, while other parameters are as in Fig. 5. 
As already explained in Sec. IIIC, the dip related to the guided mode (ii) appears approximately at the LO phonon frequency because it is stuck to the narrow frequency window $\omega_{A_{1}(L O)}<\omega<\omega_{E_{1}(L O)}$ where $\operatorname{Re} \varepsilon_{x x} / \varepsilon_{z z}<0$ and $p_{2}$ is real if the phonon damping is neglected. We notice a small but clearly seen downward shift for the sample (\#10) containing the highest carbon concentration, according to the SIMS data, as compared to the other two (see Fig. 8). As we saw in Sec. IIIC, the existence of a local impurity mode affects the position of this feature, pushing it to the lower frequency.

The mode (iii) at $\approx 770 \mathrm{~cm}^{-1}$ in Fig 8 is clearly seen only for sample \#10 with the highest $\mathrm{C}$ concentration and appears in both polarizations. Based on our calculated results presented in Sec. III, we associate it with the local vibration mode of carbon atoms substituting nitrogen in the anion sublattice sites. Let us mention in this respect that Ito et al. [4] observed a mode at $777.5 \mathrm{~cm}^{-1}$ in transmission spectra of their $\mathrm{GaN}$ :C samples, which was attributed to local vibrations of $\mathrm{C}$ atoms in the anionic sublattice of the GaN matrix.

Concerning other $p$-polarized spectral features, as we checked, the dip at $\approx 880 \mathrm{~cm}^{-1}$ in Fig 9 , also seen in the experimental spectra of Fig. 8, is related to the finite thickness of the AlGaN layer, since it disappears when $\mathrm{AlGaN}$ is considered semi-infinite in the calculations. What is rather surprising for us is that the GaN/AlGaN SPP mode is hardly seen in the experimental spectra for all three samples. One possible reason is that the thickness of the AlGaN layer, $d_{A l G a N}$, fluctuates considerably within each sample. Our calculations (performed for an ideal planar structure) show that the spectral position of this mode is quite sensitive to this thickness and can vary over a range of several tens of $\mathrm{cm}^{-1}$ depending on $d_{\text {AlGaN }}$. Thus, if the thickness (or Al/Ga fraction and therefore the dielectric function) of the AlGaN layer varies within the IR illumination area, the SPP band should be broadened and diminished in amplitude.

\section{Conclusions}

We presented a comprehensive study of a number of MOVPE grown CaN:C structures by means of Raman and IR-ATR spectroscopies. The study was motivated by the search for evidence of the presence of carbon atoms in the nitrogen sublattice of GaN. Using a simple isotope defect model, we found that such an impurity would create a local mode of estimated frequency $\omega_{l o c}=768 \mathrm{~cm}^{-1}$ while a carbon atom located in the cationic sublattice is expected to originate only a resonant mode. The local mode, theoretically, should be both Raman and IR-active, although can be too weak to be detected. Another possibility to detect the presence of electrically active carbon impurities would be 
a shift in the positions of longitudinal Raman and IR-active modes owing to the phonon-plasmon coupling.

Our non-resonant Raman spectra show the presence of three symmetry-allowed phonon modes of $\mathrm{GaN}$ and their spectral positions do not deviate considerably from the values reported by other authors (even though we observe small variations of the order of $2 \mathrm{~cm}^{-1}$ in the position of the GaN $E_{2}(h)$ mode for different samples). The absence of any observable phonon-plasmon coupling implies that the free carrier concentration is much lower than that of carbon atoms determined by SIMS. Beyond these, an extra (and much weaker) mode at $\approx 650 \mathrm{~cm}^{-1}$ has been observed, not reported in the literature for $\mathrm{GaN}$. In our opinion, it originates from the $\mathrm{AlN}$ - like $E_{2}(h)$ vibration mode of the underlying $\mathrm{AlGaN}$ alloy layer. No sign of a local vibration mode that might be associated to carbon atoms is seen, which can be too weak to be detected in Raman spectra.

In the resonant Raman scattering spectra (RRS), we observed a strong and broad photoluminescence (PL) band with superimposed multiple LO phonon peaks. The intensity and the position of the PL band clearly correlate with the carbon concentration, which implies that the exciton binding energy is slightly reduced with doping. It might be because of the screening produced by additional free carriers introduced by the doping (holes if carbon atoms are in the nitrogen sublattice or electrons if they are in the gallium sublattice). On the other hand, interpreting the higher-order LO phonon peaks in the RRS spectra in terms of the cascade model [34, 35] implies that the carbon doping does not decrease the exciton-phonon interaction, as one might expect if the impurity provided additional charge carriers. The observed decrease in the UV emission intensity with the increase of the carbon content can be explained by the onset of competing (radiative and non-radiative) impurity-mediated recombination mechanisms [31]. The obtained RRS spectroscopic data are not sufficient for definitive conclusions concerning whether any considerable fraction of the carbon atoms are in an electrically inactive state or not. The carrier concentration, even if affected by the doping, is too low to influence the Raman-active modes. With the rather high nominal carbon doping level (evaluated by SIMS) and the presence of some carbon atoms in the nitrogen sublattice (revealed by IR-ATR spectroscopy results), it can be understood by the auto-compensation effect caused by the presence of $\mathrm{C}$ atoms in both sublattices. A completely clear picture of carbon doping in $\mathrm{GaN}$ is still to emerge. Far infra-red spectroscopy, performed in the ATR configuration, shows the presence of surface polariton mode of the interface $\mathrm{GaN} /$ vacuum (appearing as a broad band at at $620-630 \mathrm{~cm}^{-1}$ owing to the finite vacuum gap between the sample surface and the ATR prism) and a guided wave mode in the GaN layer (near $737 \mathrm{~cm}^{-1}$ ). This interpretation relies on the results of the spectra modelling performed using the transfer matrix method. This mode appears in the calculated spectra only if the GaN anisotropy is taken into account, so the hexagonal crystal structure is quite important for understanding the structure of the ATR spectra [37]. With the IR-ATR spectroscopy, we have been 
able to observe a feature appearing in both $s$ - and $p$-polarizations, which we interpret as the carbon local vibration mode, based on the presented modelling results. Its position $\left(\approx 770 \mathrm{~cm}^{-1}\right)$ agrees very well with the theoretically estimated value of $\omega_{l o c}$ and the intensity correlates with the nominal $\mathrm{C}$ concentration in different samples. Additional indirect evidence of the local vibration mode effect on the ATR spectra comes from the fact that the guided wave mode is slightly downshifted in the most heavily doped sample (\#10), which is a theoretically expected effect.

We would like to point out that IR-ATR spectroscopy turns out to be the most sensitive tool (as compared to other spectroscopies applied in the present work) for this purpose, at least for the considered structures. A weak perturbation of the dielectric function of $\mathrm{GaN}: \mathrm{C}$, caused by the substitutional carbon impurity, produces a very subtle spectral feature in conventional reflection spectra (see Appendix, Fig. A1), compared to the ATR reflectivity spectra (Fig. 9). The theoretically expected GaN/AlGaN interface mode (of the Berreman type) is almost not seen in our IR-ATR spectra. We tentatively explain this fact by possible fluctuations of the thickness and/or composition of the underlying AlGaN layer within each sample but further investigation is required in order to assert this point.

\section{Acknowledgements}

This work was supported by FEDER through the COMPETE Program and by the Portuguese Foundation for Science and Technology (FCT) in the framework of the Strategic Financing UID/FIS/04650/2013 and Infineon Technologies Austria AG. 


\section{References}

[1] N. Zheludev, The life and times of the LED - a 100-year history, Nature Photonics 1, 189 (2007).

[2] M. Huber, I. Daumiller, A. Andreev, M. Silvestri, L. Knuuttila, A. Lundskog, M. Wahl, M. Kopnarski, and A. Bonanni, Characterization of AlN/AlGaN/GaN:C heterostructures grown on $\mathrm{Si}(111)$ using atom probe tomography, secondary ion mass spectrometry, and vertical currentvoltage measurements, J. Appl. Phys. 119, 125701 (2016).

[3] T. Hikosaka, T. Narita, Y. Honda, M. Yamaguchi, and N. Sawaki, Optical and electrical properties of (1-101)GaN(1-101)GaN grown on a $7^{\circ}$ off-axis (001) Si substrate, Appl. Phys. Lett. 84, 4717 (2004).

[4] S. Ito, H. Kobayashi, K. Araki, K. Suzuki, N. Sawaki, K. Yamashita, Y. Honda, and H. Amano, Resonant Raman and FTIR spectra of carbon doped GaN, Resonant Raman and FTIR spectra of carbon doped GaN, J. Cryst. Growth 414, 56 (2015).

[5] J. L. Lyons, A. Janotti, and C. G. Van der Walle, Appl. Phys. Lett. 97, 152108 (2010).

[6] M. Kuball, Raman spectroscopy of $\mathrm{GaN}, \mathrm{AlGaN}$ and $\mathrm{AlN}$ for process and growth monitoring control, Surface \& Interface Analysis 31, 987 (2001).

[7] J. Ibañez, S. Hernández, E. Alarcón-Lladó, R. Cuscó, L. Artús, S. V. Novikov, C. T. Foxon and E. Calleja, Far-infrared transmission in GaN, AlN, and AlGaN thin films grown by molecular beam epitaxy, J. Appl. Phys. 104, 033544 (2008).

[8] T. Dumelow, T. J. Parker, S. R. P. Smith and D. R. Tilley, Far-infrared spectroscopy of phonons and plasmons in semiconductor superlattices, Surface Science Reports 17, 151 (1993).

[9] W. Hayes and R. Loudon, Scattering of Light by Crystals, Courier Corporation, North Chelmsford, USA, 2012.

[10] T. Azuhata, T. Sota, K. Suzuki and S. Nakamura, Polarized Raman spectra in GaN, J. Phys.: Condens. Matter 7, L129 (1995).

[11] D. Behr, J. Wagner, J. Schneider, H. Amano, and I. Akasaki, Resonant Raman scattering in hexagonal GaN, Appl. Phys. Lett. 68, 2404 (1996).

[12] V. Yu. Davydov, A. V. Subashiev, T. S. Cheng, C. T. Foxon, I. N. Goncharuk, A. N. Smirnov, and R. V. Zolotareva, Raman scattering by surface polaritons in cubic GaN epitaxial layers, Solid State Communications 104, 397 (1997).

[13] New Semiconductor Materials. Characteristics and Properties, Electronic Archive of the Ioffe Physico-Technical Institute, Russia, http://www.ioffe.ru/SVA/NSM/

[14] V. Yu. Davydov, Yu. E. Kitaev, I. N. Goncharuk, A. N. Smirnov, J. Graul, O. Semchinova, D. Uffmann, M. B. Smirnov, A. P. Mirgorodsky, and R. A. Evarestov, Ab initio phonon dispersions of wurtzite AlN, GaN, and InN, Phys. Rev. B 58, 12899 (1998). 
[15] C. Bungaro, K. Rapcewicz, and J. Bernholc, Ab initio phonon dispersions of wurtzite AlN, GaN, and InN, Phys. Rev. B 61, 6720 (2000).

[16] G. Kaczmarczyk, A. Kaschner, A. Hoffmann, and C. Thomsen, Phys. Rev. B 61, 5353 (2000).

[17] I. M. Lifshitz, Energy spectrum and the quantum states of disordered condensed systems, Soviet Physics - Uspekhi 7, 549 (1965).

[18] M. F. Cerqueira, T. Viseu, J. Ayres de Campos, A. G. Rolo, T. de Lacerda-Aroso, F. Oliveira, I. Bogdanovic-Radovic, E. Alves, and M. I. Vasilevskiy, Raman study of insulating and conductive ZnO:(Al, Mn) thin films, Phys. Status Solidi A 212, 2345 (2015).

[19] Light Scattering in Solids II, Eds., M. Cardona and Güntherodt, Topics in Applied Physics, vol. 50, Springer, Berlin, 1982.

[20] R. Cuscó, J. Ibañez, E. Alarcón-Lladó, L. Artús, T. Yamaguchi, and Y. Nanishi, Raman scattering study of the long-wavelength longitudinal-optical-phonon-plasmon coupled modes in high-mobility InN layers, Phys. Rev. B 79, 155210 (2009).

[21] J. Ibañez, E. Tarhan, A. K. Ramdas, S. Hernández, R. Cuscó, L. Artús, R. R. Melloch, and M. Hopkinson, Direct observation of LO phonon-plasmon coupled modes in the infrared transmission spectra of n-GaAs and $n-\operatorname{In}_{x} \mathrm{Ga}_{1-\mathrm{x}}$ As epilayers, Phys. Rev. B 69, 075314 (2004).

[22] E. A. Vinogradov, Semiconductor microcavity polaritons, Physics - Uspekhi 45, 1213 (2002).

[23] K. Torii, T. Koga, T. Sota, T. Azuhata, S. F. Chichibu and S. Nakamura, Attenuated-totalreflection study on the surface phonon-polaritonin GaN, J. Phys.: Condensed Matter 12, 7041 (2000). [24] S. S. Ng, Z. Hassan, and H. Abu Hassan, Surface phonon polariton of wurtzite GaN thin film grown on c-plane sapphire substrate, Solid State Communications 145, 538 (2008).

[25] S. S. Ng, T. L. Yoon, Z. Hassan, and H. Abu Hassan, Surface and interface phonon polaritons of wurtzite GaN thin film grown on 6H-SiC substrate, Appl. Phys. Lett. 94, 241912 (2009).

[26] S. F. Shayesteh, T. Dumelow, T. J. Parker, T. I. Benushis, S. N. Ershov and M. I. Vasilevskiy, Determination of the far infrared optical constants of $n$-doped bulk $\mathrm{Cd}_{\mathrm{x}} \mathrm{Hg}_{1-\mathrm{x}} \mathrm{Te}(\mathrm{CMT})$ by dispersive Fourier transform spectroscopy, Int. J. Infr. Mm. Waves 16, 763 (1995).

[27] V. Yu. Davydov, I. N. Goncharuk, A. N. Smirnov, A. E. Nikolaev, W. V. Lundin, and A. S. Usikov, A. A. Klochikhin, J. Aderhold, J. Graul, O. Semchinova, and H. Harima, Composition dependence of optical phonon energies and Raman line broadening in hexagonal $\mathrm{Al}_{\mathrm{x}} \mathrm{Ga}_{1-\mathrm{x}} \mathrm{N}$ alloys, Phys. Rev. B 65, 125203 (2002).

[28] L. Zhang and J.-J. Shi, Surface phonon polariton modes in AlGaN thin films, Phys. Status Solidi B 246, 164 (2009).

[29] Yu. V. Bludov, Aires Ferreira, N. M. R. Peres, and M. I. Vasilevskiy, A Primer on Surface Plasmon-Polaritons in Graphene, Int. J. Mod. Phys. B 27, 1341001 (2013).

[30] D. W. Berreman, J. Opt. Soc. Am. 62, 502 (1972). 
[31] M. A. Reshchikov and H. Morkoc, Luminescence properties of defects in GaN, J. Appl. Phys. 97, $061301(2005)$

[32]M. Huber, M. Silvestri, L. Knuuttila, G. Pozzovivo, A. Andreev, A. kadashcuk, A. Bonanni and A. Lundskog, Impacto f residual carbon impurities and gallium vacancies on trapping effects in $\mathrm{AlGaN} / \mathrm{GaN}$ metal insulating semiconductor high eléctron mobility transistors, Appl. Phys. Lett. 107, $032106(2015)$

[33] M. A. Reshchilov, D. O. Demchenko, A. Usikov, H. Helava and Yu. Makarov, Carbon defects as sources of the green and yellow luminescence bands in undoped GaN, Phys. Rev. B 90, 235203 (2014)

[34] M. F. Cerqueira, M. I. Vasilevskiy, F. Oliveira, A. G. Rolo, T. Viseu, J. Ayres de Campos, E. Alves, and R. Correia, Resonant Raman scattering in $\mathrm{ZnO}: \mathrm{Mn}$ and $\mathrm{ZnO}: \mathrm{Mn}: \mathrm{Al}$ thin films grown by RF sputtering, J. Phys.: Condensed Matter 23, 334205 (2011).

[35] C. Trallero Giner, I. G. Lang, and S. T. Pavlov, Theory of Multiphonon Resonance Raman Scattering as a Function of Temperature in Polar Semiconductors, Physica Status Solidi (b) 106, 349 (1981).

[36] M. Schubert, Polarization-dependent optical parameters of arbitrarily anisotropic homogeneous layered systems, Phys. Rev. B 53, 4265 (1996).

[37] Z. G. Hu, A. B. Weerasekara, N. Dietz, A. G. U. Perera, M. Strassburg, M. H. Kane, A. Asghar, and I. T. Ferguson, Infrared optical anisotropy of diluted magnetic $\mathrm{Ga}_{1-x} \mathrm{Mn}_{x} \mathrm{~N} / c$-sapphire epilayers grown with a GaN buffer layer by metalorganic chemical vapor deposition, Phys. Rev. B 75, 205320 (2007). 
Here we present analytical expressions of the transfer $4 \times 4$ matrix method applied to electromagnetic plane waves reflected or transmitted at a stratified medium formed by isotropic or anisotropic homogeneous layers. More detailed treatments can be found in [33] and references therein. ${ }^{1}$

Consider an electromagnetic wave of angular frequency $\omega$ (wavenumber in vacuum $k_{0}=\omega / c$ ) incident on a pile of $N$ parallel faced layers between incidence (or ambient) and exit semi-infinite media. All media are linear, homogeneous and non-magnetic. The incidence and exit media are denoted by the indices $a$ and $f$, respectively. The incidence medium is isotropic with real refractive index $n_{a}$, while the layers and exit media can be isotropic or anisotropic (with principal complex refractive indices $\left.n_{x}, n_{y}, n_{z}\right)$. Let $\left(A_{p}, A_{s}\right),\left(B_{p}, B_{s}\right)$ and $\left(C_{p}, C_{s}\right)$ represent the complex amplitudes of the $p$-polarized (polarization parallel to the plane of incidence) and $s$-polarized (polarization perpendicular to the plane of incidence) of the incident, reflected and transmitted waves, respectively. Cartesian laboratory coordinate system is defined such that the $z$ axis is perpendicular to layer interfaces, the plane of incidence coincides with the $x-z$ plane and the origin is set at the interface of the incidence and the stratified media.

The four wave amplitudes inside the incidence medium and the transmitted amplitudes inside the exit medium are related by a global $4 \times 4$ transfer matrix, $T$, which describes the response of the entire multilayered structure:

$$
\left(\begin{array}{l}
A_{s} \\
B_{s} \\
A_{p} \\
B_{p}
\end{array}\right)=\mathrm{T}\left(\begin{array}{c}
C_{s} \\
0 \\
C_{p} \\
0
\end{array}\right)=\left(\begin{array}{llll}
T_{11} & T_{12} & T_{13} & T_{14} \\
T_{21} & T_{22} & T_{23} & T_{24} \\
T_{31} & T_{32} & T_{33} & T_{34} \\
T_{41} & T_{42} & T_{43} & T_{44}
\end{array}\right)\left(\begin{array}{c}
C_{s} \\
0 \\
C_{p} \\
0
\end{array}\right)
$$

Let $d_{i}$ denote the thickness of the $i$-th layer and $\mathrm{T}_{p_{i}}$ the partial transfer matrix that connects the inplane wave components at the interface at $z=z_{i}$ with those at the next interface at $z=z_{i}+d_{i}$. $\mathrm{T}_{p_{i}}$ accounts for the optical properties of a single homogeneous layer within the stratified sample. The general transfer matrix $\mathrm{T}$ is obtained from the product of all inverted matrices $\mathrm{T}_{p_{i}}^{-1}$ for each layer, as well as the inverted incidence $\left(\mathrm{L}_{\mathrm{a}}^{-1}\right)$ and exit $\left(\mathrm{L}_{\mathrm{f}}\right)$ matrices in the order of their appearance:

$$
\mathrm{T}=\mathrm{L}_{\mathrm{a}}^{-1} \prod_{i=1}^{N}\left[\mathrm{~T}_{p_{i}}\left(d_{i}\right)\right]^{-1} \mathrm{~L}_{f} .
$$

\footnotetext{
${ }^{1}$ As mentioned above, in our configuration where the $\mathrm{z}$-axis of the uniaxial GaN crystal is normal to the surface and interfaces, s- and p-polarized waves do not mix and $4 \times 4$ transfer matrix is block-diagonal, composed of two uncoupled $2 \times 2$ blocks representing each polarization. However, we prefer to present here the general formulation as it was used in our code used for numerical calculations of the spectra.
} 
The analytic expressions for the matrix $\mathrm{L}_{\mathrm{a}}^{-1}$ (isotropic medium) and for $\mathrm{L}_{\mathrm{f}}$ and $\mathrm{T}_{p_{i}}$ in the case of biaxial media whose coordinate system is oriented parallel to the laboratory coordinate axes are as follows. The $\mathrm{L}_{\mathrm{a}}^{-1}$ matrix is given by:

$$
\mathrm{L}_{\mathrm{a}}^{-1}=\frac{1}{2}\left(\begin{array}{cccc}
0 & 1 & -1 / n_{a} \cos \Phi_{a} & 0 \\
0 & 1 & 1 / n_{a} \cos \Phi_{a} & 0 \\
1 / \cos \Phi_{a} & 0 & 0 & 1 / n_{a} \\
-1 / \cos \Phi_{a} & 0 & 0 & 1 / n_{a}
\end{array}\right),
$$

Where $\Phi_{\mathrm{A}}$ is the angle of incidence and the $\mathrm{L}_{f}$ matrix is:

$$
\mathrm{L}_{f}=\frac{1}{2}\left(\begin{array}{cccc}
0 & 0 & \cos \Phi_{z} & 0 \\
1 & 0 & 0 & 0 \\
-n_{y} \cos \Phi_{y} & 0 & 0 & 0 \\
0 & 0 & n_{x} & 0
\end{array}\right)
$$

where the angle $\Phi_{j}$ (index $j$ stands for $y$ or $z$ ) is obtained applying Snell's law

$$
\cos \Phi_{j}=\sqrt{1-\left[\left(n_{a} / n_{j}\right) \sin \Phi_{a}\right]^{2}} .
$$

The partial transfer matrix $\left(\mathrm{T}_{p}\right)$ for a layer of thickness $d$ is given by:

$$
\mathrm{T}_{p}=\left(\begin{array}{cccc}
\cos \left(k_{p}\right) & 0 & 0 & i \frac{N_{x z}}{n_{x}^{2}} \sin \left(k_{p}\right) \\
0 & \cos \left(k_{s}\right) & -i \frac{1}{N_{y y}} \sin \left(k_{s}\right) & 0 \\
0 & -i N_{y y} \sin \left(k_{s}\right) & \cos \left(k_{s}\right) & 0 \\
i \frac{n_{x}^{2}}{N_{x z}} \sin \left(k_{p}\right) & 0 & 0 & \cos \left(k_{p}\right)
\end{array}\right)
$$

where

$$
\begin{gathered}
k_{p}=k_{0} d N_{x z} ; \quad k_{s}=k_{0} d N_{y y}, \\
N_{i j}=n_{i} \cos \Phi_{j},
\end{gathered}
$$

$k_{\mathrm{o}}=\omega / \mathrm{c}$ is the wavevector in vacuum, $\cos \Phi_{j}$ is given by (A5) (index $i$ stands for $x$ or $y$ and index $j$ stands for $y$ or $z$ ) and $n_{\mathrm{i}}$ is the corresponding refractive index. Notice that due to the symmetry properties of the partial transfer matrix $\left[\mathrm{T}_{p}(d)\right]^{-1}=\mathrm{T}_{p}(-d)$. Expressions for the matrices in the case of media with higher symmetry are attained by imposing $n_{x}=n_{y}=n_{z}$ (isotropic medium) or $n_{x}=n_{y} \neq n_{z}$ (uniaxial medium) in expressions (A4) to (A8).

The reflecting properties of the anisotropic system may be characterized by four reflection coefficients $r_{s s}, r_{s p}, r_{p s}$ and $r_{p p}$, that are related to the elements of the general transfer matrix: 


$$
\begin{aligned}
& r_{s s}=\left(\frac{B_{s}}{A_{s}}\right)_{A_{p}=0}=\frac{T_{21} T_{33}-T_{23} T_{31}}{T_{11} T_{33}-T_{13} T_{31}} ; \\
& r_{s p}=\left(\frac{B_{p}}{A_{s}}\right)_{A_{p}=0}=\frac{T_{41} T_{33}-T_{43} T_{31}}{T_{11} T_{33}-T_{13} T_{31}} ; \\
& r_{p s}=\left(\frac{B_{s}}{A_{p}}\right)_{A_{s}=0}=\frac{T_{11} T_{23}-T_{21} T_{13}}{T_{11} T_{33}-T_{13} T_{31}} ; \\
& r_{p p}=\left(\frac{B_{p}}{A_{p}}\right)_{A_{s}=0}=\frac{T_{11} T_{43}-T_{41} T_{13}}{T_{11} T_{33}-T_{13} T_{31}}
\end{aligned}
$$

The first and second subscripts of the coefficients denote the polarization of the incoming and reflected waves, respectively. For example, $r_{s p}$ is the amplitude of the wave reflected into the $p$ polarization when the incident wave is s-polarized. If no analyzer is used (light arriving at the detector may have both $s$ and $p$ polarizations), the reflectance for incident $s$ - or $p$-polarized light can be calculated according to, respectively:

$$
\begin{aligned}
& R_{s}=\left|r_{s s}\right|^{2}+\left|r_{s p}\right|^{2}, \\
& R_{p}=\left|r_{p p}\right|^{2}+\left|r_{p s}\right|^{2} .
\end{aligned}
$$

The above relations can be applied to either ATR (in which case the "entrance" medium is the prism, the angle of incidence exceeds that of total internal reflection and a vacuum gap is introduced between the prism and the structure) or conventional reflection (when the "entrance" medium is vacuum). For comparison with ATR spectra presented in the main text, conventional reflectance spectra of the whole structure are shown in Fig. 1A.

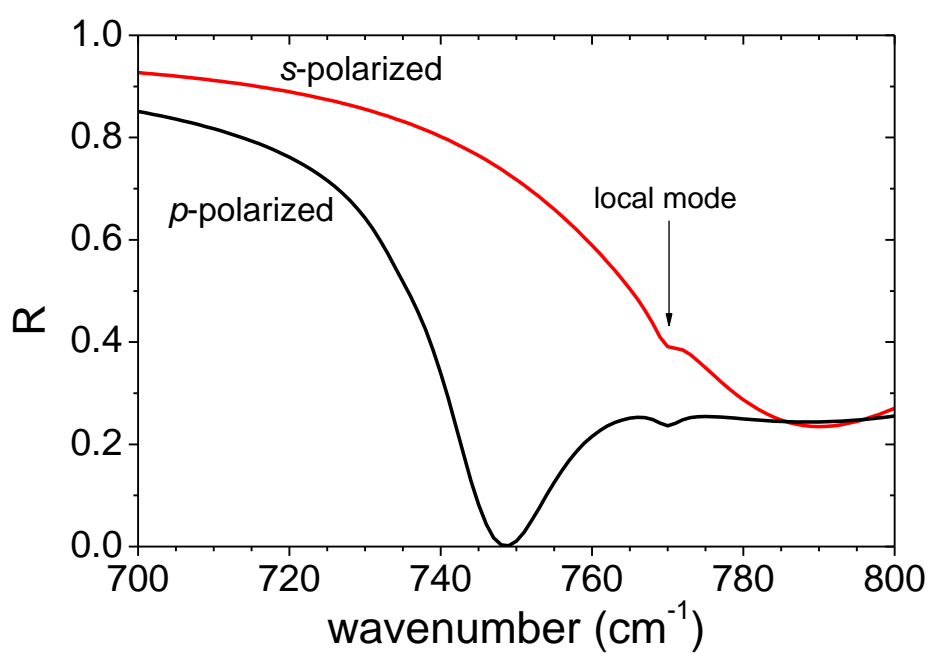

Figure A1. Calculated $s$ - and $p$-polarized conventional oblique incidence $\left(45^{\circ}\right)$ reflection spectra of $\mathrm{GaN} / \mathrm{AlGaN} / \mathrm{AlN} / \mathrm{Si}$ structure. All parameters are as in Fig. 9. 
These general relations can be simplified for the case of a single layer of a uniaxial material with the $c$ axis normal to the surface, cladded by two isotropic media with dielectric constants $\varepsilon_{1}$ and $\varepsilon_{3}$. In this case $r_{s p}=r_{p s}=0$ and it suffices to consider a $2 \times 2$ transfer matrix for each polarization. It is convenient to transform from the general complex amplitudes $A, B$ and $C$ to the amplitudes of the relevant electric and magnetic field components. For $p$ polarization we have:

$$
H_{y}=H_{y}^{(i)}\left(1+\hat{r}^{p}\right)=\sqrt{\varepsilon_{1}}\left(A_{p}+B_{p}\right) ; \quad E_{x}=\frac{k_{1 z}}{\sqrt{\varepsilon_{1}} k_{1}} H_{y}^{(i)}\left(1-\hat{r}^{p}\right)=\cos \Phi_{a}\left(A_{p}-B_{p}\right)
$$

for the cladding medium 1 (note that here $\cos \Phi_{a}=k_{1 z} / k_{1}$, where $k_{1}$ and $k_{1 z}$ are the wave vector and its $z$ component in medium 1, respectively) and

$$
H_{y}^{(t)}=\hat{t}^{p} H_{y}^{(i)}=\sqrt{\varepsilon_{3}} C_{p} ; \quad E_{x}^{(t)}=\frac{k_{3 z}}{\sqrt{\varepsilon_{3}} k_{3}} H_{y}^{(t)}=\cos \Phi_{f} C_{p}, \quad \cos \Phi_{f}=k_{3 z} / k_{3}
$$

for the cladding medium 3. Here $\hat{r}^{p}=r_{p p}$ and $\hat{t}^{p}=H_{y}^{(t)} / H_{y}^{(i)}$ are the Fresnel coefficients. The $2 \times 2$ transfer matrix corresponding to the basis $\left(H_{y}, E_{x}\right)^{T}$ is:

$$
\tilde{\mathrm{T}}_{p}=\left(\begin{array}{cc}
\cos \left(p_{2} d\right) & -i \frac{\varepsilon_{x x} \omega}{c p_{2}} \sin \left(p_{2} d\right) \\
-i \frac{c p_{2}}{\varepsilon_{x x} \omega} \sin \left(p_{2} d\right) & \cos \left(p_{2} d\right)
\end{array}\right),
$$

where $p_{2} \equiv \sqrt{\varepsilon_{x x} / \varepsilon_{z z}} k_{2 z}$. It yields the field components in the medium 1 (at $z=0$ ) in terms of those in medium 3 (at $z=d$ ). The explicit expressions for the Fresnel coefficients are given in the main text [Eqs. (8)]. 